\title{
A Case of Severe Hyperammonemic Encephalopathy Caused by Urinary Tract Infection in Obstructive Uropathy
}

\section{Bo Gyung Mun, M.D. Joo Hoon Lee, M.D., Ph.D. Young Seo Park, M.D., Ph.D Jiwon Jung, M.D. \\ Department of Pediatrics, Asan Medical Center Children's Hospital, University of Ulsan College of Medicine, Seoul, Republic of Korea}

Corresponding author:

Jiwon Jung, M.D.

Department of Pediatrics, Asan Medical Center Children's Hospital, University of Ulsan College of Medicine, 88, Olympic-ro 43-Gil, Songpa-Gu, Seoul, 05505, Korea

Tel: +82-2-3010-0034

Fax: +82-2-3010-3356

E-mail:jiwonjung0322@gmail.com

Received: 3 September 2021

Revised: 18 October 2021

Accepted: 16 November 2021
This is an open-access article distributed under the terms of the Creative Commons Attribution Non-Commercial License (http:// creativecommons.org/licenses/by-nc/4.0/) which permits unrestricted non-commercial use, distribution, and reproduction in any medium, provided the original work is properly cited.

Copyright (C) 2021 The Korean Society of Pediatric Nephrology
Hyperammonemia is mainly caused by diseases related to liver failure. However, there are also non-hepatic causes of hyperammonemia, such as urinary tract infection (UTI) due to urease-producing organisms. Urease production by these bacteria induces a hydrolysis of urinary urea into ammonia that can cross the urothelial cell membrane and diffuse into blood vessels, leading to hyperammonemia. Delayed diagnosis and treatment of hyperammonemia can lead to lethal encephalopathy that can cause brain damage and life-threatening conditions. In the presence of obstructive uropathy, UTI by urease-producing bacteria can lead to more severe hyperammonemia due to enhanced resorption of ammonia into the systemic circulation. In this report, we present a case of acute severe hyperamm. onemic encephalopathy leading to brain death due to accumulation of ammonia in blood caused by Morganella morganii UTI in a 10-year-old girl with cloacal anomaly, causing obstructive uropathy even after multiple corrections.

Key words: Cloaca, Gram-negative bacteria, Morganella morganii, Sepsis-Associated Encephalopathy, Ureteral Obstruction

\section{Introduction}

Hyperammonemia can cause severe encephalopathy, permanent neurologic deficit, or even death if not treated properly and immediately ${ }^{1)}$. It is considered a medical emergency due to its high mortality rate and severe neurologic sequelae in case of survival. Therefore, early diagnosis and management are crucial. Hyperammonemia is associated with several conditions, such as acute liver failure, inborn errors of metabolism (e.g., urea cycle defects including ornithine transcarbamylase [OTC] deficiency or N-Acetylglutamate synthetase deficiency), and urease-producing bacterial infections from the urinary tract with or without obstructive uropathy ${ }^{2)}$. Urinary stasis can induce bacterial overgrowth, and urinary tract infection (UTI) caused by urease-producing bacteria can predispose a patient to develop hyperammonemia. Marked urinary stasis can increase the exposure of bladder mucosa to urine, and with the increase in urine ammonia in obstructive uropathy, it may increase diffusion of ammonium into nearby blood vessels and directly into systemic circulation ${ }^{3)}$.

Cloacal malformation is a rare congenital anomaly with its incidence estimated to be 1 per 50,000 live births ${ }^{4}$. This condition can cause obstructive 
uropathy immediately after birth or even after undergoing staged surgical correction accompanied with retention of urine in cloacal structures, bladder dysfunction, renal dysplasia with or without obstruction, vesicoureteral reflux (VUR), and aberrant course of urethra with stricture.

In this case report, we present a case of pediatric acute hyperammonemic encephalopathy due to UTI by ureaseproducing bacteria superimposed on cloacal anomaly related obstructive uropathy, which eventually led to brain death.

\section{Case report}

A 10-year-old girl was admitted to our pediatric emergency room with acute symptoms of vomiting, irritability, and mental change that started 11 hours before the visit.

She was born with multiple congenital anomalies including cloacal anomaly, and underwent surgical procedures involving descending colostomy, vaginostomy, and cystostomy immediately after birth. At the age of 1 year, total vaginectomy and total hysterectomy were performed due to severe adhesion and non-dissectability during the Peña operation of the gastrointestinal tract. Neourethral formation was performed but urination was mainly maintained via cystostomy, and partly with a neourethra until she gained proper urethral urination at the age of 3 years. Although proximal urethral stricture was suspected, the patient showed tolerable voiding without residual urine; therefore, cystostomy was safely removed and she maintained self-voiding via urethra.

Regarding the upper urinary tract, she had dysplastic change of the left kidney with multiple cysts and severe hydroureteronephrosis with cortical thinning in the right kidney at birth. She also had bilateral VUR (right grade III, and left grade V). She was repeatedly hospitalized due to recurrent UTIs until the age of 2; Escherichia coli was detected as the causative bacteria at one instance, Pseudomonas aeruginosa was detected in two instances, and Actinobacter baumannii was detected once in the urine cultures. Persistent right hydroureteronephrosis and newly detected left hydroureteronephrosis were observed on follow-up imaging at the age of 9 years, when the glomerular filtration rate (GFR) decreased to $30 \mathrm{ml} / \mathrm{min} / 1.73 \mathrm{~m}^{2}$ (BUN
$26 \mathrm{mg} / \mathrm{dL}$, serum creatinine $1.32 \mathrm{mg} / \mathrm{dL}$ ). The patient was regularly followed up in the outpatient clinic.

A day prior to the visit to the emergency department, the patient developed abdominal discomfort during urination. Irritability with recurrent nausea and vomiting followed, and 3 hours later, she was anuric. The patient presented to our pediatric emergency room (PER) 8 hours after the onset of anuria with persistent irritability.

Laboratory findings at the time of admission are listed in Table 1. The white blood cell count was $31,300 / \mu \mathrm{L}$ (neutrophil count of $88.8 \%$ ). Acute kidney injury was found; BUN $51 \mathrm{mg} / \mathrm{dL}$, serum creatinine $1.99 \mathrm{mg} / \mathrm{dL}$, uric acid $6.4 \mathrm{mg} / \mathrm{dL}$, and eGFR $25 \mathrm{ml} / \mathrm{min} / 1.73 \mathrm{~m}^{2}$. The fractional excretion of sodium was calculated to be $27.7 \%$. The urine sampled after intravenous rehydration showed specific gravity of 1.015; WBC, 11-20/high-power field (HPF); and RBC, 11-20/HPF. The patient was febrile with no obvious focus; thus, intravenous antibiotics targeted for possible UTI were started. The presence of Morganella morganii, a urease-producing bacteria, was later confirmed twice from initial and follow-up urine cultures.

At the time of visit, the patient was screaming and uncooperative. Her Glasgow coma scale (GCS) score was 11 points at the time of arrival (Eye opening, 4; Movement, 5; Verbal 2), and there were no specific clinical findings such as significant abdominal distension or tenderness on physical examination. Four hours after admission, GCS score worsened to 9 (E2 M5 V2) and further worsened to a score of 8 (E2 M4 V2) after 6 hours. Cerebrospinal fluid (CSF) tapping was performed with an opening pressure of 17.5 $\mathrm{cm} \mathrm{H}_{2} \mathrm{O}$ with no remarkable results from the analysis (Table 1). Initial brain computed tomography (CT) scan showed no signs of acute intracranial hemorrhage or overt infarction, hydrocephalus, brain edema (Fig. 1A). The patient further deteriorated after few hours with no response to localized pain, and generalized rigidity (GCS 4, semicoma). Plasma ammonia level was checked to be $417 \mu \mathrm{mol} /$ $\mathrm{L}$ at this point. Initially, as the exact etiology for acute hyperammonemia was unknown, sodium benzoate was administered in case of possible late onset urea cycle disease. Even though the patient's condition was less likely caused by late onset inborn error of metabolism, we decided to instill sodium benzoate while waiting for preparation of continuous renal replacement therapy (CRRT), ruling out 
the slightest possibility of late onset metabolic etiology at the same time. The patient was transferred to the intensive care unit (ICU) afterwards for CRRT to remove ammonia and for close monitoring of neurologic status. As oliguria was persistent during PER stay, the residual urine volume was checked at this point, revealing $342 \mathrm{cc}$ with evident

\section{Table 1. Laboratory analysis on admission}

\begin{tabular}{|c|c|}
\hline Laboratory parameter & Values \\
\hline WBC $\left(/ \mathrm{mm}^{3}\right)$ & 31,300 \\
\hline Neutrophil (\%) & 88.8 \\
\hline Lymphocyte (\%) & 8.4 \\
\hline Hemoglobin (g/dL) & 14.6 \\
\hline Platelet (/ $\mathrm{LL})$ & 399,000 \\
\hline BUN/Cr (mg/dL) & $51 / 1.99$ \\
\hline AST/ALT (IU/L) & $24 / 11$ \\
\hline ALP (IU/L) & 267 \\
\hline Protein/Albumin (g/dL) & $8.1 / 4.6$ \\
\hline Uric acid (mg/dL) & 6.4 \\
\hline Amylase/Lipase (U/L) & $126 / 24$ \\
\hline Total bilirubin (mg/dL) & 0.7 \\
\hline Serum glucose & 297 \\
\hline Sodium (mmol/L) & 144 \\
\hline Potassium (mmol/L) & 3.8 \\
\hline Chloride (mmol/L) & 110 \\
\hline Magnesium (mg/dL) & 2.72 \\
\hline CRP $(\mathrm{mg} / \mathrm{dL})$ & 0.1 \\
\hline $\mathrm{eGFR}(\mathrm{ml} / \mathrm{min} / \mathrm{m} 2)$ & 25 \\
\hline Ammonia ( $\mu \mathrm{mol} / \mathrm{L})$ & 417 \\
\hline B-ketone (mmol/L) & 0.1 \\
\hline \multicolumn{2}{|l|}{ Urinalysis } \\
\hline Appearance & Light turbid \\
\hline SG & 1.015 \\
\hline $\mathrm{pH}$ & 8.0 \\
\hline WBC (/HPF) & $11-20$ \\
\hline RBC (/HPF) & $11-20$ \\
\hline Albumin & + \\
\hline Glucose & - \\
\hline Nitrite & - \\
\hline \multicolumn{2}{|c|}{ Cerebrospinal fluid analysis } \\
\hline Color & colorless \\
\hline Turbidity & clear \\
\hline $\mathrm{RBC}(/ \mu \mathrm{L})$ & 1 \\
\hline 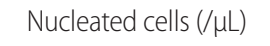 & 2 \\
\hline Protein (mg/dL) & 24.6 \\
\hline Glucose (mg/dL) & 148 \\
\hline
\end{tabular}

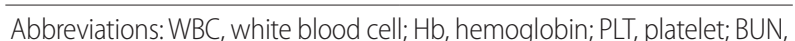
blood urea nitrogen; $\mathrm{Cr}$, creatinine; AST, aspartate aminotransferase; ALT, alanine aminotransferase; LDH, lactate dehydrogenase; CRP, C-reactive protein; eGFR, estimated GFR; HPF, high-power field; SG, specific gravity. bladder distension. Needle cystostomy was immediately performed at the bedside to relieve urinary retention; a total of 1,050 mL turbid fluid was drained. Urine ammonia level was $2,606.5 \mu \mathrm{mol} / \mathrm{L}$. At the same time, CRRT was initiated; continuous veno-venous hemodiafiltration (CVVHDF) mode was used with blood flow rate (BFR) of $110 \mathrm{ml} / \mathrm{min}$, replacement fluid flow rate (RFFR) of $500 \mathrm{ml} / \mathrm{hr}$ and dialy. sate flow rate (DFR) of $3,000 \mathrm{ml} / \mathrm{hr}$ for maximal efficiency in lowering ammonia level. After CRRT initiation, the ammonia level was dropped to $106 \mu \mathrm{mol} / \mathrm{L}$ within 4 hours but unfortunately, her mental status deteriorated (GCS 3, E1 V1 M1). The patient showed Kussmaul's breathing pattern followed by desaturation and ceasing of spontaneous respiration, necessitating intubation and mechanical ventila-

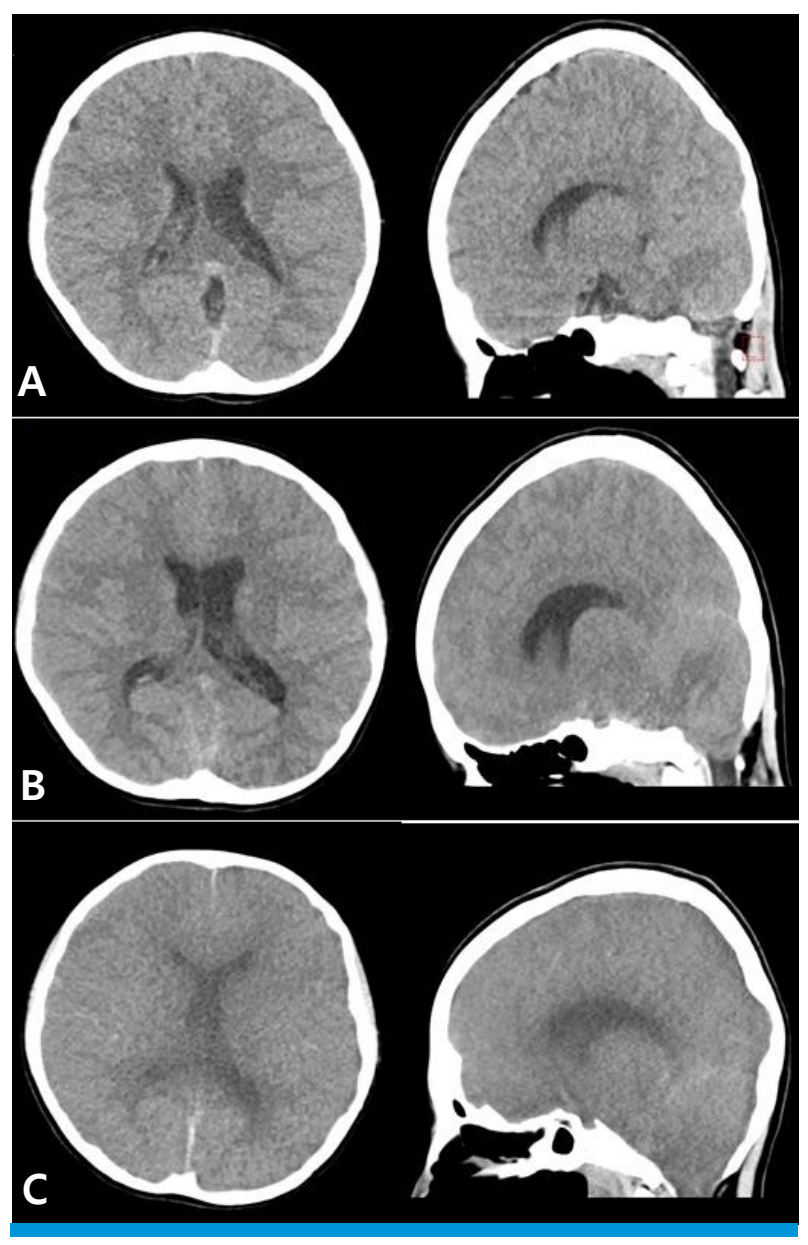

Fig. 1 Series of brain computed tomography scans through the hospital course of the patient; on the day of admission with nonspecific findings (A), at hospital day 4 showing diffuse sulcal effacement in the brain, compressed brainstem, suspected swelling of the cerebellum and mild tonsillar herniation (B), and at hospital day 6 with deterioration of brain edema with overt herniation $(C)$. 
tion. Follow-up brain CT scan revealed mild sulcal effacement and swelling of the cerebellum and mild tonsillar herniation (Fig. 1B). Electroencephalography (EEG) showed diffusely suppressed background activity over the whole hemisphere. Eight hours after admission to the ICU, bilateral pupillary reflexes were absent and the patient was comatose (GCS 3).

To rule out the possibility of late onset urea cycle disease, diagnostic examinations including plasma acylcarnitine, urine organic acid, serum amino acid, and genetic evaluation including whole exome sequencing, chromosomal microarray analysis, and evaluation for OTC mutation were performed. However, there were no significant findings to suggest metabolic disease.

On hospital day 2 , body temperature dropped below $36^{\circ} \mathrm{C}$ and there was no elicitable anal tone. Comatose mental status persisted with fixed dilated pupils and absent brainstem-related light reflex, corneal reflex, oculocephalic reflex, vestibulo-ocular reflex, cilio-spinal reflex, gag reflex, and cough reflex. Follow-up brain CT (Fig. 1C) confirmed severe cerebral edema. EEG findings corresponded to the diagnosis of brain death. After confirmation of brain death, the life-sustaining treatment was halted in accordance with the guardian's intention, and the patient died.

\section{Discussion}

This report presents a rare case of lethal hyperammonemic encephalopathy in a patient with urease-producing UTI who had obstructive uropathy caused by cloacal anomaly. Urinary stasis due to obstruction made the patient vulnerable to frequent UTIs, and ammonia produced by urease-producing bacteria diffused into the systemic circulation resulting in hyperammonemia.

Hyperammonemia is usually defined as a plasma level of ammonia that is above $80 \mu \mathrm{mol} / \mathrm{L}$ in infants (up to 1 month of age) and above $55 \mu \mathrm{mol} / \mathrm{L}$ in older children ${ }^{5}$. Ammonia is produced in the gastrointestinal tract as a metabolic product of protein digestion and bacterial metabolism. Then, it enters the urea cycle in the liver in which it is metabolized into non-toxic urea. Therefore, the most common cause of hyperammonemia in pediatric patients is liver failure, and rarely, inherited metabolic diseases affecting the urea cycle.
Other possible causes of hyperammonemia should be considered, including UTI by urease-producing organisms, dehydration, high protein intake, gastrointestinal bleeding, parenteral nutrition, bone marrow transplantation, or medications. When hyperammonemia is not managed properly and promptly, encephalopathy with symptoms of mental change and seizures can ensue, leading to permanent brain injury. Thus, early diagnosis and prompt treatment is important to prevent neurological sequelae and avoid fatal complications.

The patient in our case had no underlying liver disease and was too old to be suspected of hyperammonemic crisis due to inborn errors of metabolism. Serologic tests and genetic analysis revealed no metabolic derangements in the urea cycle. The severe hyperammonemia in our patient was attributable to urease-producing Morganella morganii UTI. When the urinary tract is infected with urease-producing bacteria, urease hydrolyzes urea in the urine to ammonium ions(NH4+), which elevate urinary $\mathrm{pH}^{6}$. In highly alkaline urine, the ammonium molecule is transformed into ammonia (NH3) which is a liposoluble free base that can cross cell membranes. A combination of alkalized urine from excessive production of bacterial urease and urinary stasis due to obstructive uropathy can lead to the enhanced diffusion of ammonia through the bladder wall into the perivesical circulation. Moreover, most venous blood from the bladder bypasses the portal circulation and drains directly into the hypogastric vein and inferior vena cava, which accelerates the accumulation of ammonia in the systemic circulation.

Although there are several case reports of hyperammonemia in adults due to non-hepatic causes such as urinary obstruction $^{7}$ and UTIs caused by urease-producing bacteria $^{8,9)}$, there is a paucity of pediatric cases. Nonetheless, there have been few reports of pediatric patients who had UTI by urea splitting organisms, and coincidentally had underlying obstructive uropathy with sudden abnormal rise in serum ammonia. In one case, an 8-month-old boy with known hydronephrosis was admitted with symptoms of vomiting, irritability, poor feeding and convulsions ${ }^{10)}$. The patient's initial ammonia level was $299 \mu \mathrm{mol} / \mathrm{L}$, which increased further $(453 \mu \mathrm{mol} / \mathrm{L})$ despite sodium benzoate loading. Proteus mirabilis detected from the urine, was the cause of hyperammonemia in this case; immediate per- 
www.chikd.org

cutaneous nephrostomy led to normalization of ammonia, although neurologic sequelae persisted afterwards. In another case, a previously healthy 10 -month-old boy presented with lethargy and unresponsiveness following gross hematuria, and dysuria ${ }^{111}$. Serum ammonia level at admission was $185 \mu \mathrm{mol} / \mathrm{L}$. He had dilatation of both renal collecting systems, and a cystogram revealed a large trabeculated bladder with a filling defect by ureterocele. Percutaneous bilateral nephrostomy and removal of ureterocele was performed. Blood ammonia quickly dropped and Proteus mirabilis was later detected as the causative bacteria for UTI. Fortunately, the patient recovered without any neurologic sequelae.

The present case of a 10-year-old girl with acute hyperammonemic encephalopathy due to UTI caused by ureaseproducing bacteria Morganella morganii with obstructive uropathy was the first such case reported in our country, and the first to be associated with "Morganella morganii" to the best of our knowledge. Pre-existing congenital cloacal anomaly and urinary stasis due to both anatomic and functional defects led to obstructive uropathy and vulnerability to recurrent UTI. Urine stagnation in combination with urease-producing bacterial infection caused noxious ammonia accumulation directly into the systemic circulation leading to hyperammonemic encephalopathy. Patient had a catastrophic clinical course despite maximal support to lower the ammonia level right after the detection of hyperammonemia.

In summary, we report a case of pediatric hyperammonemic encephalopathy due to UTI by urease-producing bacteria in a patient with obstructive uropathy related to cloacal malformation, which eventually led to hyperammonemic encephalopathy. Our findings suggest that it is important to have a high index of suspicion for hyperammonemia in pediatric patients with underlying urologic obstructive lesions and provide prompt assessment and management for early correction of hyperammonemia.

\section{Acknowledgements}

This study was approved by the institutional review board (IRB), and the consent was waived due to the nature of the retrospective study [IRB number 2021-0979].

\section{Conflicts of interest}

No potential conflict of interest relevant to this article was reported.

\section{ORCID}

Bo Gyung Mun https://orcid.org/0000-0002-0555-7778 Joo Hoon Lee https://orcid.org/0000-0001-8010-3605 Young Seo Park https://orcid.org/0000-0001-7653-2036 Jiwon Jung https://orcid.org/0000-0001-5358-7966

\section{References}

1. Burton BK. Urea cycle disorders. Clin Liver Dis 2000;4:815-30.

2. Savy N, Brossier D, Brunel-Guitton C, Ducharme-Crevier L, Du Pont-Thibodeau G, Jouvet P. Acute pediatric hyperammonemia: current diagnosis and management strategies. Hepatic Med Evid Res 2018:10:105-15.

3. Kaveggia FF, Thompson JS, Schafer EC, Fischer JL, Taylor RJ. Hyperammonemic encephalopathy in urinary diversion with ureasplitting urinary tract infection. Arch Intern Med 1990;150:238992.

4. Warne S, Chitty LS, Wilcox DT. Prenatal diagnosis of cloacal anomalies. BJU Int 2002;89:78-81.

5. Ozanne B, Nelson J, Cousineau J, Lambert M, Phan V, Mitchell G, et al. Threshold for toxicity from hyperammonemia in critically ill children. J Hepatol 2012;56:123-8.

6. Braude A.I., Siemienski J. Role of bacterial urease in experimental pyelonephritis. J Bacteriol 1960;80:171-9.

7. Kiyokawa H, Igawa Y, Nishizawa O. Hyperammonaemic encephalopathy associated with retention of urine in multiple large diverticula. BJU Int 2001;88:122-3.

8. Goda T, Watanabe K, Kobayashi J, Nagai Y, Ohara N, Takahashi D. A case of hyperammonemia with obstructive urinary tract infection by urease-producing bacteria. Rinsho Shinkeigaku (Clin Neurol) 2017;57:130-3.

9. Emura M, Tsuchihashi K, Shimizu Y, Kanamaru S, Matoba S, Ito N. A Case of Hyperammonemia Caused by Urinary Tract Infection Due to Urease-Producing Bacteria. Hinyokika Kiyo. Acta Urologica Japonica 2016;62:421-5.

10. McEwan P, Simpson D, Kirk JM, Barr DG, McKenzie KJ. Short report: Hyperammonaemia in critically ill septic infants. Arch Dis Child 2001;84:512-3.

11. Sinha B, Gonzalez R. Hyperammonemia in a boy with obstructive ureterocele and proteus infection. J Urol 1984;131:330-1. 